\title{
Une écologie perceptive hors de l'humain
}

\section{A perceptive ecology outside of humans}

\author{
Edwige Armand ${ }^{1}$, Frédérick Garcia ${ }^{2}$ \\ ${ }^{1}$ Laboratoire LARA-SEPPIA, INP Purpan (edwige.armand@gmail.com) \\ ${ }^{2}$ MIAT, INRA, Centre Occitanie-Toulouse (frederick.garcia@inra.fr)
}

RÉSUMÉ. À partir d'une installation art-science autour de la perception du végétal notamment au niveau sonore, nous interrogerons plus largement notre relation au vivant. La question de l'impossibilité de comprendre l'alterité des mondes perceptifs de chaque organisme est un des axes qui est articulé dans cet article autour des pôles de production de connaissances que sont la science et les arts. Le monde végétal nous invite à revoir nos catégories de pensées usuelles telles que la mémoire, l'individualité, le temps, l'identité, la séparabilité.

ABSTRACT. Through an art-science installation based on the perception of plants, particularly in terms of sound, we will examine our relationship with living organisms. The question of the impossibility of understanding the alterity of the perceptual worlds of each organism is one of the axes that is articulated in this article around, the poles of knowledge production that are science and the arts. The vegetable world invites us to review our usual categories of mind such as memory, individuality, time, identity, separability.

MOTS-CLÉS. Perception, perspectivisme, végétal, son, art et science, êtres vivants.

KEYWORDS. Perception, perspectivism, plant, sound, art and science, living beings.

Nous présenterons dans un premier temps une réflexion sur nos mécanismes perceptifs avec un regard critique d'une science qui peine à aborder la complexité du vivant. On poursuivra alors sur nos recherches artistiques et scientifiques liées à l'étude du vivant et plus particulièrement du végétal, et nous finirons en présentant une pièce réalisée en collaboration liant les arts et les sciences.

Comme l'a exprimé H.Bergson ${ }^{1}$ l'être humain a construit son intelligence sur une matérialité aux propriétés statiques, se clôturant dans un mode perceptif qui s'accorde avec les propriétés de sa matérialité. Cela a permis un découpage de la temporalité qu'il nomme les instants $t$ et nous permet de créer la causalité en faisant des arrêts virtuels qu'il s'agit ensuite de lier artificiellement grâce à une conception linéaire de la temporalité. Partant de cette critique de la temporalité, Bergson nous dit que nous sommes incapables de comprendre ainsi le mouvant et le temps qui lui ne s'arrête pas. Mais cette division et organisation du temps seront les modalités perceptives par lesquelles la science pourra comprendre son environnement et avoir une maîtrise par une performativité sur le monde qu'elle observe et participe à produire. Ce découpage de la temporalité grâce à une perception photographique s'accorde avec le langage et la logique qui permettent par une suspension des détails et du mouvant de penser le monde selon des états fixes et dans une réalité pratique, mais incomplète. Grâce à ces cristallisations provisoires, l'homme est capable de se détacher du présent immédiat. La cristallisation de ces formes de temps permet de retenir et projeter un temps passé pour prévoir un futur non advenu. Nous sommes des prédicateurs, nous dit le neuroscientifique Alain Berthoz. Pour prévoir un temps non fait, nous projetons ce que nous connaissons sur ce qui n'est pas encore réalisé. Nous ne pouvons que prévoir ce que sera l'évènement, qui découle de nos attentes motrices et cognitives déçues ou comblées. Notre capacité de rétention et de protention, liée à notre survie, fait que la qualité de l'évènement est bien une

\footnotetext{
${ }^{1}$ Henri Bergson, L'évolution créatrice, Paris, PUF, 2013, p. 165.
} 
éternelle comparaison sur du connu, permise par la mémoire et la conception d'une temporalité pensée comme linéaire. C'est parce que nous lions les événements entre eux comme une succession que nous pouvons comparer un état passé à un état présent qui dépendra de ce passé, mais qui dépend de ce présent en retour. Ce tour de passe-passe ${ }^{2}$ temporel nous a permis de construire un monde de qualité et de fait viable pour nous et nous le vivons comme une évidence. Par exemple, si l'on prend le contraste de qualité, le jaune est tout ce que le violet n'est pas et vice versa. Le jaune dépend du violet et le violet dépend du jaune. En soi, la couleur ne possède pas de qualité propre. Idem pour la musique. Si nous prenons une chanson de Yma Sumac, phénomène vocal à la tessiture couvrant plus de quatre octaves, l'aigu que l'on entend est relatif au grave que nous venons de percevoir, lui-même relatif à l'aigu que nous venons à l'instant de mémoriser.

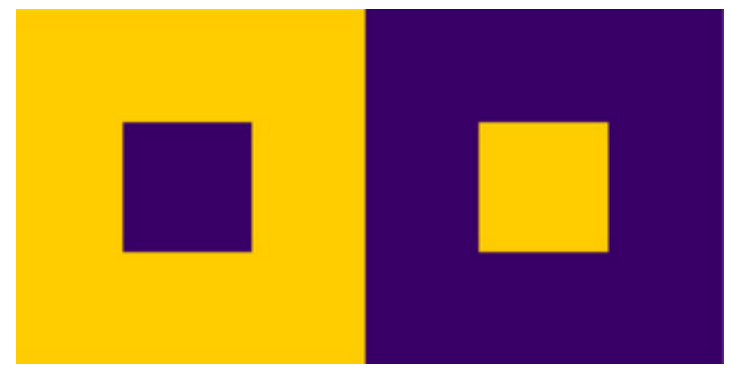

L'élaboration de la qualité du présent en outre, s'articule également à la pensée articulée à la langue. Les mots permettent de fixer des états qui sans eux seraient absents, permettant les généralités et la pensée symbolique, matrone de l'abstraction. La pensée articulée à la langue ne peut comprendre la totalité inanalysée dont parlera Peirce, mais découpe le monde aux contours définis. Ces arrêts virtuels, que nous expérimentons grâce à une langue, une logique et une conception de la temporalité, seront ceux par quoi la science pourra s'assurer une maîtrise d'une compréhension physique par une maîtrise du visible et de l'intelligible. Imitant des processus modélisés par une conception schématisée d'un réel et d'une calculabilité et rationalité imposées, s'intriquant à une temporalité arbitrairement conçue, la science a réussi à performer un réel, notamment en ce qui concerne les faits physiques, mesurables grâce à leurs qualités extensives.

La science a permis de nombreuses avancées en nous accordant sur du sensible et du perceptible, mais selon Bergson, nous excellons dans des sciences aux propriétés statiques et où le temps peut être spatialisé. En revanche, le vivant, qui est de l'ordre du mouvant et de l'irreproductible échappe selon lui, à cette temporalité figée et à nos mesures.

Nous avons créé des mesures et des normes afin de comprendre par imitation les processus naturels sous l'ordre d'une mesure spatiale. Mais comme l'exprime Hannah Arendt $^{3}$, imiter n'est pas comprendre, l'imitation ne nous assure aucune certitude, ni aucune compréhension concernant le monde vivant et notre environnement. Selon cette philosophe, l'homme, ne pouvant comprendre le « pourquoi » des phénomènes physiques, s'exerça à imiter les processus naturels sans pouvoir en sonder la finalité et réduisit le monde à l'utilité qu'il pouvait en extraire, notamment en le réduisant à des calculs. Ainsi, selon Arendt, nous regarderions le monde sous l'angle de l'utilité, de la logique, des mesures et nous aurions évacué la finalité pour nous intéresser aux moyens. Notre science, nous dit-elle, est non seulement réduite à l'utilité, mais nous ne saurions saisir la complexité d'un monde qui n'est pas à notre mesure. Pourtant, depuis la Renaissance et encore aujourd'hui, s'est progressivement construite une science que nous pourrions aujourd'hui qualifier

\footnotetext{
${ }^{2}$ Gilles Deleuze, Différence et répétition, Paris, PUF, 2011.

${ }^{3}$ Hannah Arendt, Condition de l'homme moderne, Paris, Pocket, 2012, p. 353.
} 
d'anthropocentrique et de gnoséocentrique, faisant le succès du progrès technique et médical que nous lui concédons. Notre science d'une efficacité imparable reste une pensée du calcul, du rendement, de l'hyperlogique et de l'hypertechnologique que nous avons perfectionné au cours des siècles, délaissant d'autres formes de pensées possibles et formes de connaissances, notamment celles abordant les qualités vivantes, inextensives qui sont propres à une temporalité en mouvement.

L'environnement façonne l'homme et sa culture, mais l'homme a façonné à son tour son environnement, le transformant jusqu'au point de ne plus être que confronté qu'à lui-même et aux idéologies, aux mythes qu'il y a placés. La civilisation occidentale et son hégémonisme du visible font que la multiplicité des façons de penser et de sentir le monde tendent à se standardiser. Pourtant certaines cultures, comme celle des Indiens Jivaros Achuar ${ }^{4}$, les runa-puma, peuvent nous apporter grâce à leur pensée animiste une connaissance qui pourrait bien nous ouvrir à d'autres horizons perceptifs. En dépassant la pensée symbolique intrinsèquement humaine et en nous ouvrant à une pensée sémiotique élargie, on peut concevoir que d'autres espèces sont capables de représentation iconique ou encore indicielle comme l'a développé Eduardo Khon ${ }^{5}$. Nous pourrions comprendre que d'autres manières de représenter ou percevoir le monde existent, en adoptant un point de vue autre, non utilitaire, ni sous le mode du calculatoire, du maitrisable, de la domination ou de l'exploitation.

Outre les civilisations Amazoniennes, les arts révèlent des mondes de perceptions qui pourraient encore nous garantir ce besoin d'ouverture perceptive, d'ouverture encore à d'autres modes de connaissances, afin que l'homme ne s'enferme pas dans une unique formalité de l'être et d'une production uniformalisée d'un phénomène à vivre et observer. Les arts échappent partiellement à la pensée articulée à la langue et présentent des signes dénotés qu'il s'agit de comprendre comme autant de mondes à voir. Les arts réouvrent l'impensé et l'in-vu, l'inconnu qui n'est pas lié à nos mécanismes perceptifs usuels. Les arts déjouent nos attentes sensori-motrices et nous permettent d'appréhender l'évènement dans ce qu'il a de plus étranger à l'homme, de plus brutal dira Peirce. Comme l'énonce Ortega y Gasset ${ }^{6}$, les artistes ne nous montrent pas l'humain, mais nous donnent accès à ce que le monde a d'inhumain, c'est-à-dire ce qui n'est pas soumis aux normes de notre langue, et à l'utile. Si les artistes présentent des formes de réalités singulières, les scientifiques vont également chercher à dépasser les formes de connaissances admises, mais avec des méthodologies normées et des injonctions d'utilitarisme de plus en plus fortes. C'est en liant ces deux domaines d'exploration du réel, que nous avons cherché à créer d'autres représentations du vivant.

Notre recherche artistique et scientifique soulève indirectement ces questionnements sous l'angle particulier du monde végétal, en nous interrogeant sur ce que celui-ci questionne de nos limites perceptives et cognitives. Le monde végétal demande aujourd'hui à chacun de penser l'altérité dans ce qu'elle a de plus exclusive. Le végétal nous oblige à nous départir de nos modalités perceptives et nous force à imaginer ce que pourraient bien être les perceptions et les sensations d'un organisme qui a développé d'autres stratégies évolutives. Nos recherches communes demandent comme l'indique J.M Schaeffer' de faire preuve d'un peu d'humilité en nous mettant au défi de penser audelà ou en deçà de l'homme, objectifs en fait quasi-impossibles.

\footnotetext{
${ }^{4}$ Philippe Descola, Par-delà nature et culture, Paris, Gallimard, 2005.

${ }^{5}$ Eduardo Kohn, Comment pensent les forêts, Paris, Zones Sensibles, 2017.

${ }^{6}$ José Ortega y Gasset, La déshumanisation de l'art, Paris, Allia, 2011, p. 33.

${ }^{7}$ Jean-Marie Schaeffer, La fin de l'exception humaine, Paris, Gallimard, 2007, p. 140.
} 
Les recherches que nous allons aborder, et qui sont menées à l'INRA de Toulouse par Frédérick Garcia sont entre l'Intelligence Artificielle et la biologie végétale et visent à explorer des phénomènes qui relèvent de ce que l'on peut qualifier aujourd'hui de cognition végétale. Nous nous intéressons ainsi aux phénomènes de mémoire, d'apprentissage, d'anticipation chez les plantes, et nous sommes tout particulièrement sensibles à la question de la perception sonore chez les plantes, ainsi qu'à ce qui pourrait prendre la forme d'une signalisation électrique en leur sein. Ces questions passionnantes sont explorées depuis quelques années maintenant par des chercheurs comme Monica Gagliano, Stefano Mancuso, Frantisek Baluska, Paco Calvo Garzon, à l'orée des disciplines biologie, écologie, philosophie et sciences cognitives.

Ces recherches remettent en cause notre compréhension de nombreux concepts, telle la mémoire, qui ne serait plus uniquement située dans des réseaux neuronaux puisque nous savons que les plantes sont capables d'apprentissage et de reconnaissance sans pour autant posséder des neurones. Mais aussi l'individu, la conscience...qui ne sont pas des catégories de pensée adaptées pour penser le végétal. Les notions comme «la mémoire », la «conscience » «l'identité » «l'individu » sont des termes que nous utilisons fréquemment sans être capables ni de les définir précisément, ni de les situer. En effet : «où se situe l'information dans un corps qui ne cesse de se métamorphoser, dans des cellules qui ne cessent de mourir et de muter ? »demande le chercheur en neurobiologie Alain Prochiantz titulaire de la chaire «Processus morphogénétiques » du Collège de France. Selon lui, l'ensemble des concepts humains devraient non seulement s'entendre au pluriel, mais également s'écrire dans une langue qui tienne compte du mouvant, du virtuel. Le vivant n'est pas soumis au calcul et à la logique qui appartiennent à notre système temporel, comme nous l'avons développé en amont, nous ne pouvons le réduire à notre mesure. C'est l'ensemble de nos idées, de l'ontologie qui cristallise des formes rigides de pensées qui ne peuvent être à la hauteur d'un temps vivant qui se forme et se déforme simultanément, qui émerge et qui jaillit tout le temps. Ainsi nous dit Alain Prochiantz à propos de l'embryogenèse silencieuse (destruction-création de l'ensemble de nos cellules en un an), et reprenant ainsi le célèbre paradoxe de l'identité :

«Et une dernière question pointe passablement angoissante. Si nous changeons au niveau moléculaire et cellulaire, si le génome est instable comme le sont certains réseaux de neurones, si nous sommes le siège de renouvellements aussi massifs que je viens de le proposer, et dont nous devons accepter qu'ils sont à l'origine de notre capacité d'adaptation au niveau individuel, de notre individuation, comment pouvons-nous être certain d'être nous-mêmes $?^{8}{ }^{»}$

Notre perception est avant tout statique, inerte selon Bergson et nous permet de découper dans le flux des évènements des états qui nous assurent la notion d'existence, des choses et objets nous renvoyant des images discernables et qui nous permettent en retour de nous situer. Seulement cette conception séparatrice, divisée, ne nous permet pas de concevoir le mouvant et le vivant. Notre capacité de nous externaliser et de projeter dès lors notre internalité sur le monde nous a permis de nous concevoir en tant qu'être individué mais en nous séparant d'un environnement dans lequel nous sommes immergés, et où nous nous pensons pourtant différenciés et étrangers. Si les notions d'individu, d'être, d'état, de mémoire, de conscience nous ont permis de concevoir un genre d'humanité en Occident, ces notions sembleraient peut-être inadaptées pour penser le monde du vivant et en particulier du végétal. C'est un monde perçu sous la forme de l'arrêté, des limites et du discerné qu'il nous a fallu remettre en question, car dans le végétal, la notion d'individu, d'espèce sont des notions impropres à leur mode de reproduction.

\footnotetext{
${ }^{8}$ Alain Prochiantz, Qu'est-ce que le vivant ?, Paris, Seuil, 2012, p. 67.
} 
La question qui traverse nos recherches artistiques et scientifiques est bien l'impossibilité de penser l'autre, de se départir d'un anthropocentrisme. Nous sommes des êtres aux perceptions couplées à des représentations cartésiennes et euclidiennes, représentations liées à la pensée articulée à la langue, rendant difficile la possibilité de penser le multiple simultanément et le systémique. Nous avons donc conçu, dans une démarche art-science pleinement assumée, une installation nommée «Qui parle donc ? », qui reprend une formule de Bruno Latour pour savoir qui fait parler en réalité les faits qui pourraient bien être muets. Nous avons tenté de traduire cette question en terme plastique par la notion de bruit, d'instabilité, de réseau, par des objets sonores, lumineux et visuels fluides, dynamiques, distribués, etc.

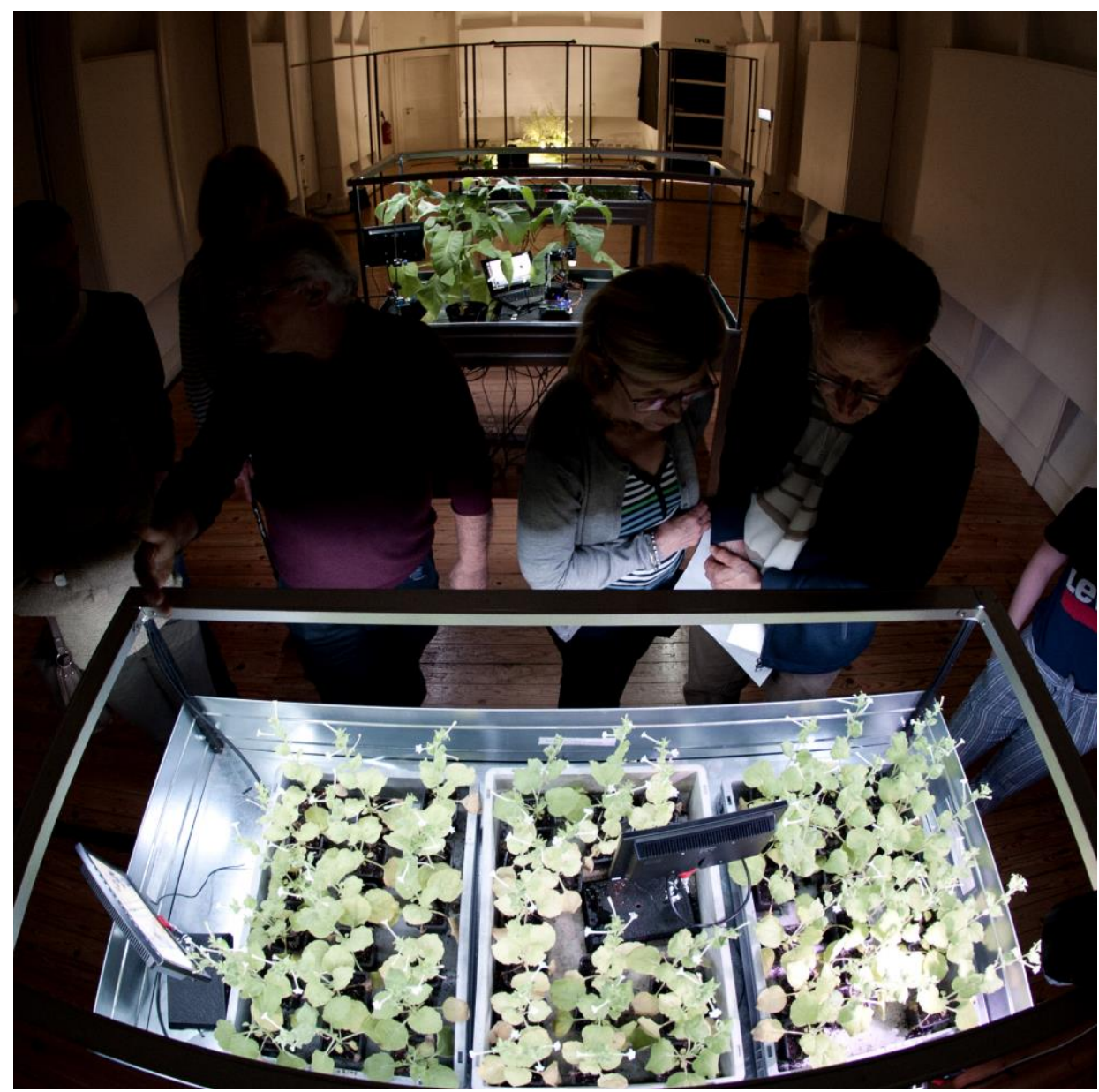

Vue générale d'une des deux pièces de l'exposition. Crédit Photo : Ariane Ruebrecht

Nous avons imaginé cette installation avec deux espaces, l'un nourri par la démarche scientifique, l'autre par la démarche artistique, pour bien donner à voir au public ces deux façons de voir le monde.

Dans la partie scientifique, à partir d'une sélection d'expérimentations menées en laboratoire, nous avons tenté d'explorer la sensibilité du végétal vis-à-vis du phénomène sonore principalement, pour le décliner sous d'autres formes comme par exemple la sensibilité au vent. Ainsi ce que nous avons voulu explorer, faire découvrir, possiblement rendre réel au public, c'est tout d'abord la capacité des plantes à entendre des sons, des vibrations sonores.

Pour la première, l'idée était de montrer des recherches actuelles qui cherchent à comprendre dans quelle mesure les plantes réagissent en temps réel en leur sein par une signalisation électrique à des stimulations extérieures, comme le son et comme le vent. 


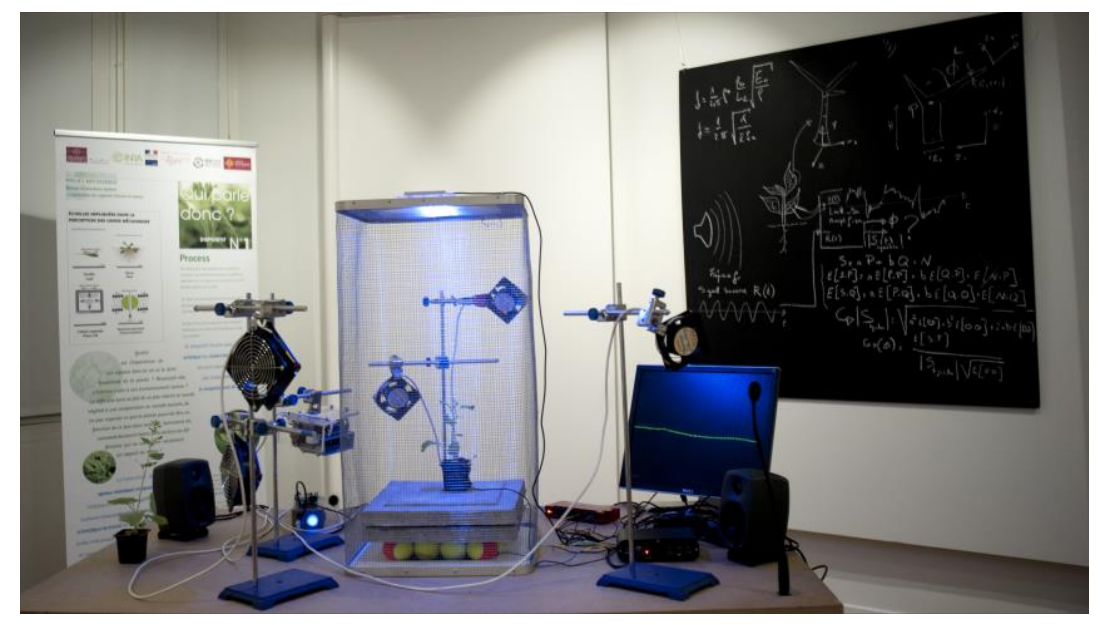

Pour la seconde, il s'agissait de reprendre une expérimentation récente semblant montrer que le système racinaire d'une plante peut reconnaître le son de l'eau et s'orienter en conséquence.

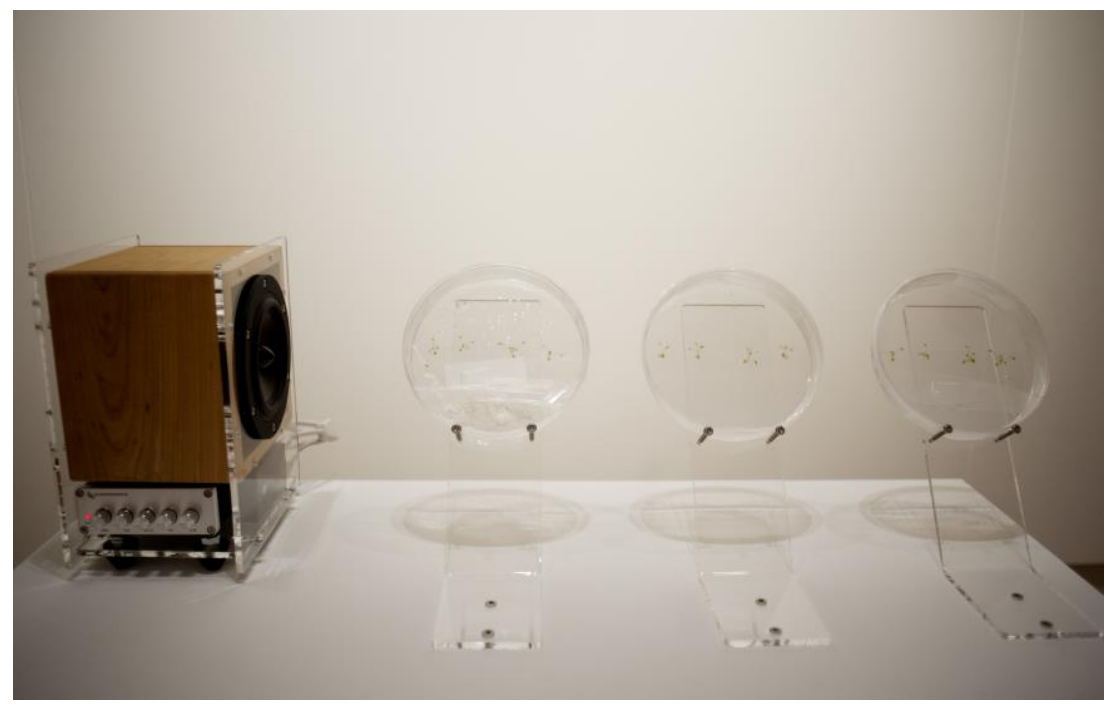

Pour ces deux expériences, nous avons souhaité les replacer, un peu à l'encontre de ce qui se fait souvent dans nos laboratoires, dans un contexte plus naturel, et cela s'est traduit par un choix d'environnements sonores naturel, grâce aux magnifiques enregistrements de Fernand Deroussen, audionaturaliste.

Avant de rentrer dans la partie artistique, nous proposons un « espace entre », murmure des sons que nous connaissons dans une évidence commune. D'abord invité à écouter des sons prélevés de différents environnements, le spectateur est en terrain connu dans le sens où ce qu'il entend et voit est familier. 

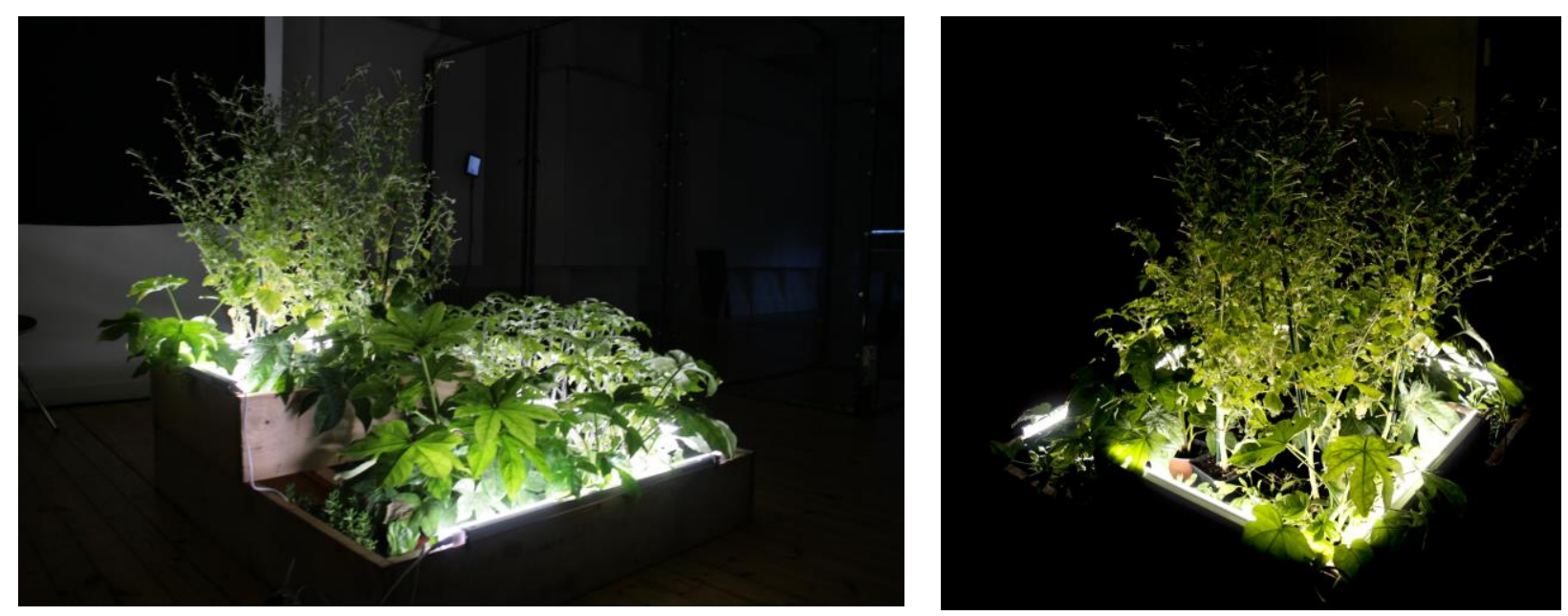

Nous l'invitons ensuite dans la partie artistique de «Qui parle donc ? » où l'idée est de rendre le spectateur et l'homme étranger.

Un sas qui fait écho à la cage de Faraday doit être franchi dans lequel il est filmé et où son image est morcelée, différée et se retranscrit devant un écran face à lui. Nous avons voulu exprimer le fait que le monde végétal non seulement est soumis à un mode perceptif non différencié, où il perçoit tout en même temps (pas d'œil, ni bouche, ni oreille), où rien n'est centralisé, mais qu'il possède également une temporalité qui n'est pas la même que celle de l'homme.
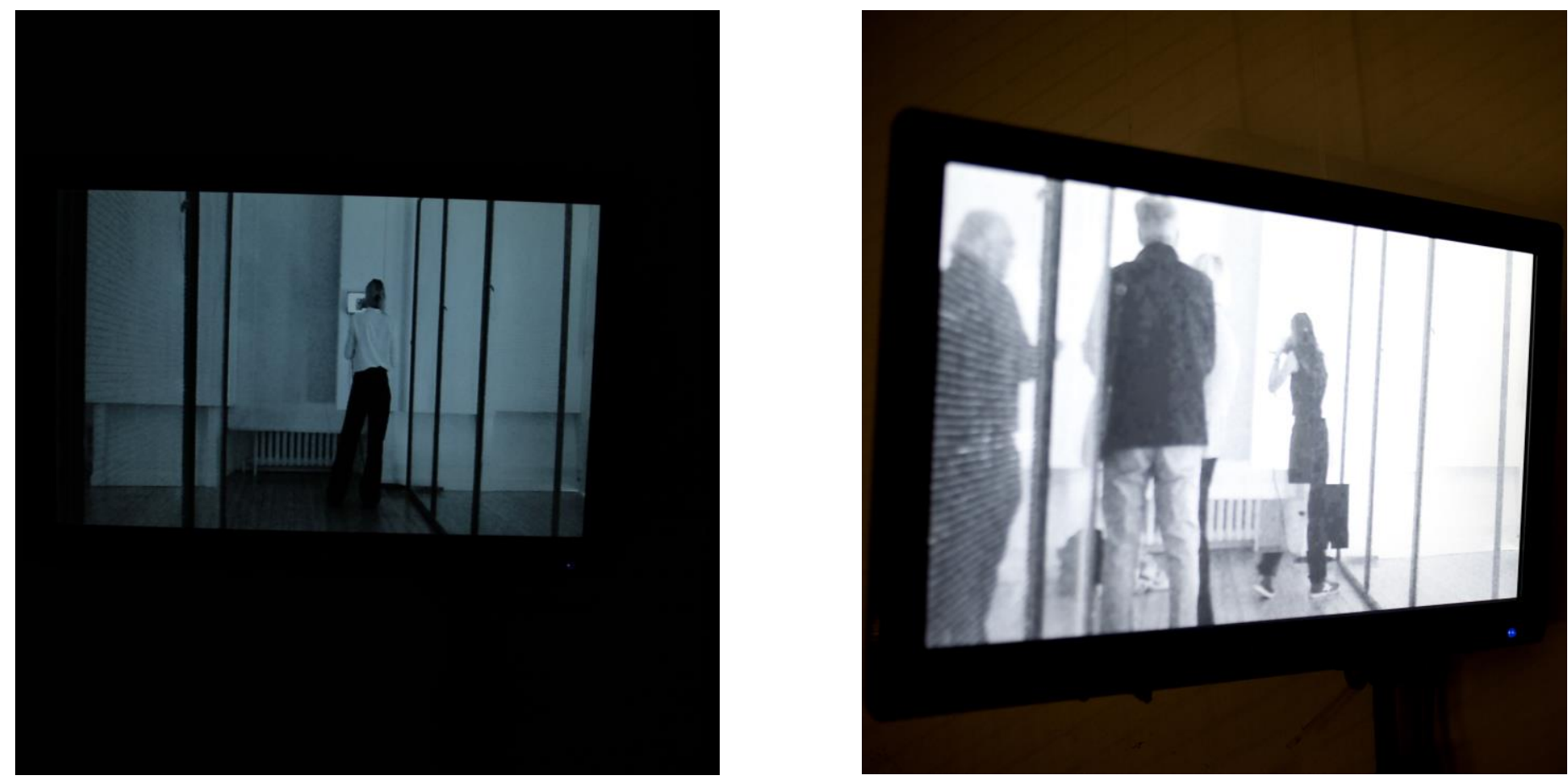

L'ensemble de la pièce dure 45 minutes avec 7 scénarios possibles où s'articulent 3 états.

Le premier état est celui de l'artificiel-naturel, où s'hybride dans des gros plans (un son perçu à l'échelle de la feuille) des sons naturels prélevés, grossis et mixés avec des sons artificiels. Un jeu de lumière y est associé.

Dans cet état le spectateur est invité à regarder un premier bac. Ici, ce sont les plantes qui l'observent et qui trackent son regard. Nous avons voulu inverser les rôles et insister sur l'idée que quelque part l'homme avec ses instruments de mesures et d'observations ne fait que s'observer lui- 
même et que nos outils ne font que reproduire un système perceptif nous empêchant de voir et comprendre l'autre.

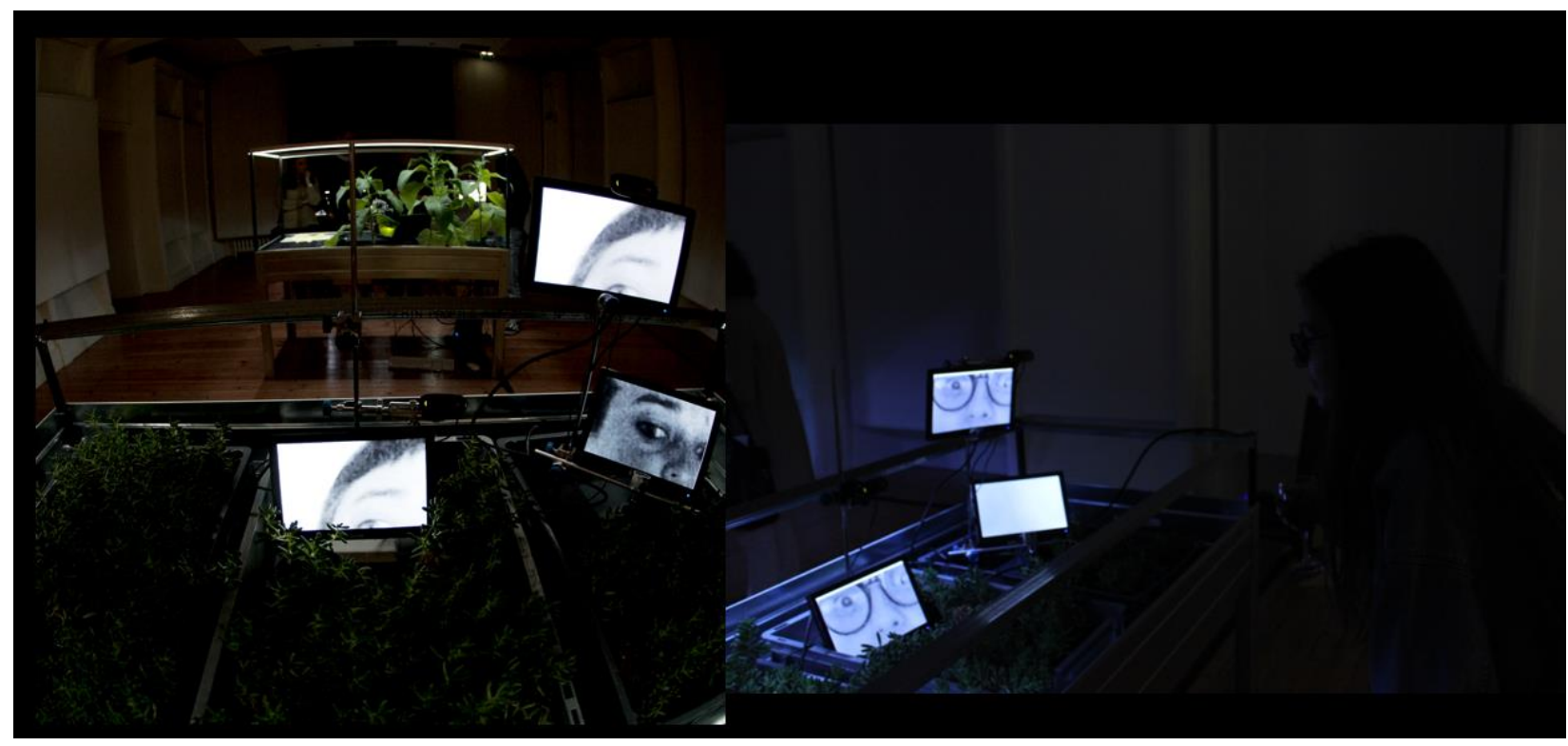

Dans un second bac, nous avons voulu jouer sur les sensations du végétal à un niveau macro et micro. Le végétal n'est pas soumis à la même échelle que nous et ici, c'est un monde micro qui est donné à voir. Le végétal perçoit un monde physique à une échelle bien plus microscopique que nous. Un microscope motorisé inspecte les feuilles, montre une échelle différente de la nôtre.

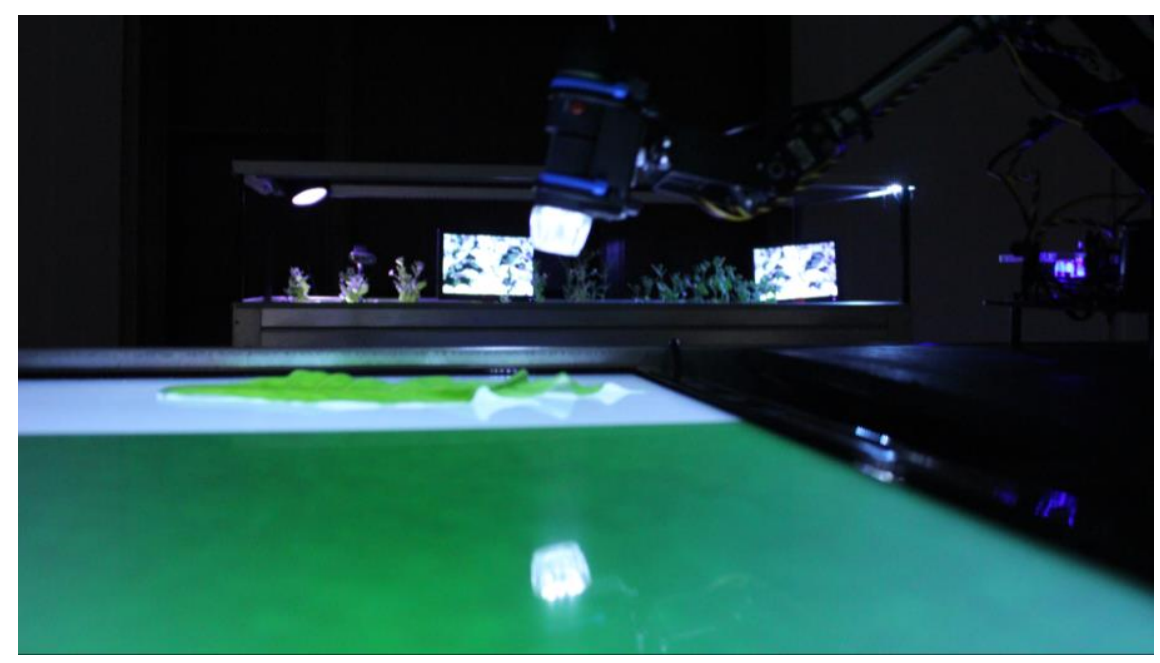




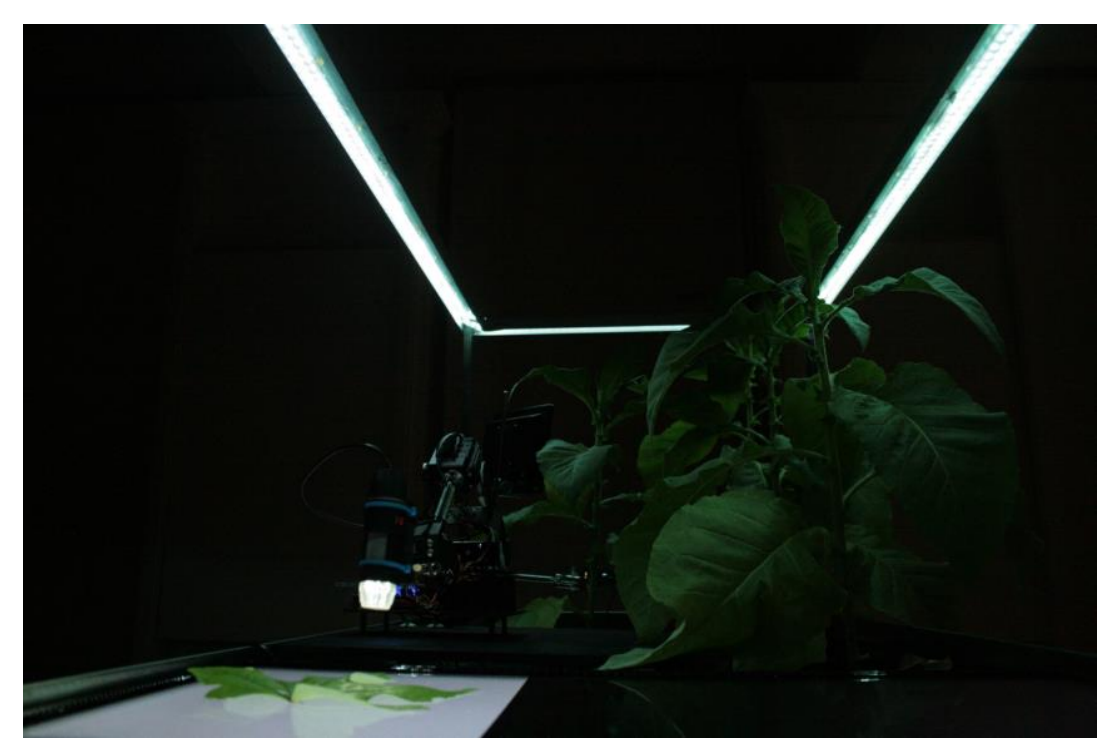

Dans le troisième bac, nous avons souhaité représenter l'idée que le végétal ne possède pas la même temporalité que l'homme. Son temps est plus étiré et long. Le végétal se meut en permanence, mais de manière imperceptible pour nous. Nous avons donc réalisé des chronophotographies (toutes les 5 minutes) que nous compilons et accélérons ensuite, et cela dans une diffusion en temps réel.

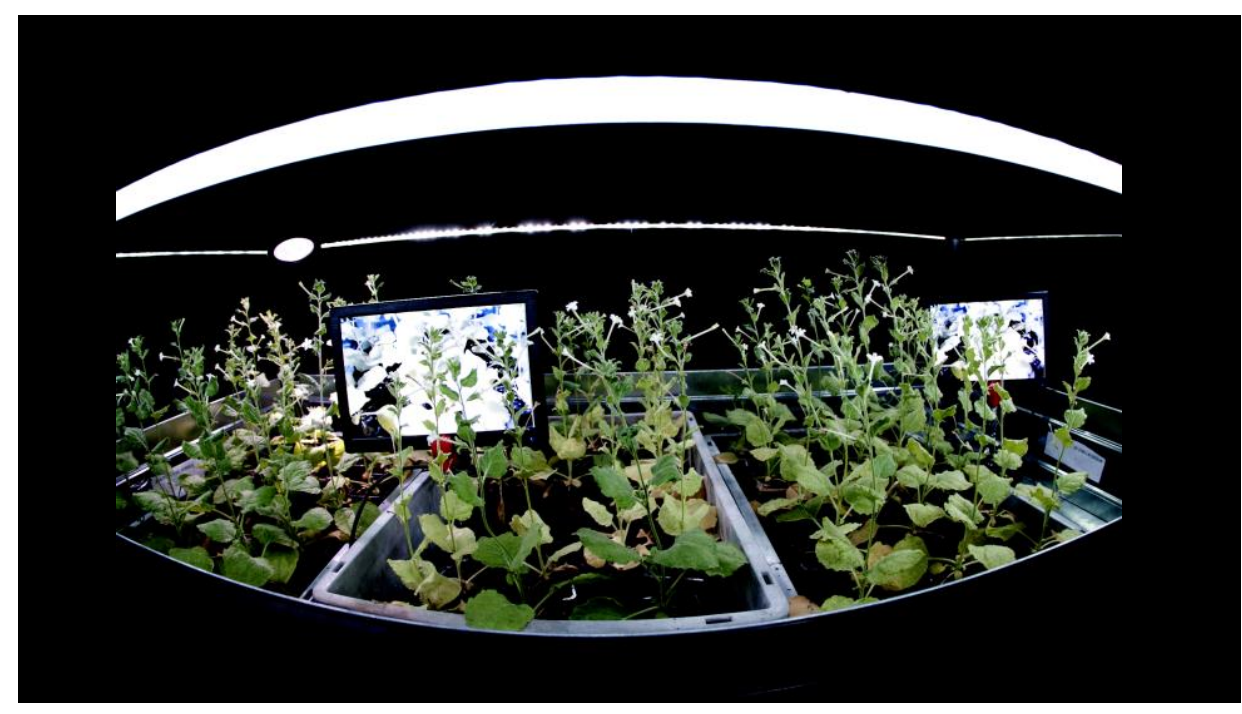

Le deuxième état perceptible est placé sous le signe de la surveillance et du son. La surveillance comme critique de la mesure et de la vision normée sous contrôle de l'être humain. Chaque écran peut être permuté, c'est-à-dire que les images du bac 3 peuvent à tout moment s'implanter dans le bac 1, et diffusent des images du spectateur prises à son insu ainsi qu'un son d'alarme et d'état d'alerte. Cette forme plastique est venue du fait que non seulement aucun organe chez la plante n'est différencié et qu'elle peut percevoir tout en même temps, mais aussi que le végétal possède une connaissance endogène de son activité. Autrement dit, une plante a connaissance via transmission d'informations de ce qui se passe au niveau de chaque feuille, des racines...

La multiplicité des caméras, des écrans était aussi inscrite dans une volonté de montrer le végétal par une multiplicité de points de vue qui remet en question la notion d'ontologie, pour s'approcher davantage de la notion de pluri-ontologie développée par Eduardo Viveiros de Castro ${ }^{9}$. Cet

\footnotetext{
${ }^{9}$ Eduardo Viveiros de Castro, Métaphysiques Cannibales, Paris, PUF, 2017.
} 
anthropologue explique que pour les peuples amazoniens, tout est doué de subjectivité et que chaque organisme se considère comme humain. L'épistémologie amazonienne est inversée par rapport à la nôtre. Il explique que ce ne sont pas les hommes qui se sont éloignés de la nature, mais la nature qui s'est éloignée de l'homme. Que ce qui n'est pas sujet ne peut pas être connu. Pour eux chaque espèce est humaine, mais chacun exclut l'autre dans son humanité. Il explique entre autres, et c'est ce qui nous intéresse, cette notion de perspectivisme en prenant l'exemple du sang. Pour nous le sang est du sang tandis que pour le jaguar le sang est de la bière de manioc. Les propriétés ontologiques dépendent de la perspective et des signes que nous y associons en fonction de notre système perceptif, de notre environnement. Nous avons voulu dans un certain sens soulever l'idée de Jakob von Uexküll et la notion d'Umwelt. Chaque organisme est enfermé dans un monde perceptif de signification utile et nécessaire, mais qui en dehors de son système perceptif développé pourrait bien être absent. Ainsi toute information n'aurait de sens que pour un organisme qui lui donne sens. Cette idée a non seulement été conduite par la multiplicité d'échelle, de point de vue, mais aussi au niveau du son et plus particulièrement de la notion de bruit.

Le troisième état est lié à cette notion qui va perturber non seulement les images, mais aussi le son et les lumières. Ainsi les écrans se mettent à diffuser de la neige blanche ou grise, le son devient bruit et la lumière se saccade dans une lumière blanche et parfois éblouissante. Nous sommes partis de Lacan qui dit que «le réel est le bruit où l'on peut tout entendre ». Tout ce que nous voyons, entendons, fait donc signe et sens pour nous, mais cela est une forme de réalité qui ne pourrait se plaquer sur d'autres organismes. Le son que nous entendons n'a certainement pas la même teneur physique pour le végétal qui ne perçoit pas les mêmes signaux. Le signal, l'information existe dans un monde humain, mais ne pourrait bien avoir aucune existence pour d'autres organismes, tout comme le fait que nous, êtres humains, n'existons certainement pas pour le monde végétal.

Ainsi à certains moments, c'est l'ensemble de la pièce visuelle, lumineuse et sonore qui est soumise au bruit, insinuant cette idée que nous pourrions bien être étrangers au monde végétal et que ce que nous percevons pourrait être sans sens pour ces organismes complexes et échappant encore aux mesures humaines.

Au-delà d'une création, il est aussi question d'un partage de connaissance humaine pour nous essayer à penser l'Altérité qui nous fait face et qui nous demande d'adopter une multiplicité de points de vue, en nous invitant au perspectivisme selon les termes d'Eduardo Viveiros de Castro. Nous avons essayé et essayons encore d'approcher une représentation qui tiendrait compte de la « différence sans rapport au même et à l'identique ${ }^{10}$ », de l'absolue différence dans ce qu'elle a de plus irreprésentable. C'est en esquissant, cherchant humblement d'autres paradigmes qui ne soient pas contraints par le cartésianisme que nous avons réalisé cette pièce, qui se voulait être à michemin de notre humanité et du monde végétal par une approche autant sensible que scientifique. C'est un paysage sonore, lumineux et visuel, pensé à partir du monde végétal, mais qui ne pourrait être finalement qu'un bruit sans signe dans lequel chacun y cherche le sens qu'il veut.

\section{Auteurs de Qui Parle Donc ? :}

Edwige Armand, Adelin Barbacci, Thierry Besche, Fernand Deroussen (audio-naturaliste), Yves Duthen, Frédérick Garcia, Mathieu Chamagne (programmation), Cédric Cambon (lumière)

\footnotetext{
${ }^{10}$ Gilles Deleuze, Différence et répétition, Paris, PUF, 2001, p. 37.
} 
Conception robot et cage de Faraday :

Théo De La Hogue \& Pol Perez 